Journal of Mathematics and Statistics 6 (3): 294-299, 2010

ISSN 1549-3644

(C) 2010 Science Publications

\title{
Proof of Analytic Extension Theorem for Zeta Function Using Abel Transformation and Euler Product
}

\author{
Mbaitiga Zacharie \\ Department of Media Information Engineering, \\ Okinawa National College of Technology, \\ 905 Henoko, Nago, 905-2192, Okinawa, Japan
}

\begin{abstract}
Problem statement: In the prime number the Riemann zeta function is unquestionable and undisputable one of the most important questions in mathematics whose many researchers are still trying to find answer to some unsolved problems such as Riemann Hypothesis. In this study we proposed a new method that proves the analytic extension theorem for zeta function. Approach: Abel transformation was used to prove that the extension theorem is true for the real part of the complex variable that is strictly greater than one and consequently provides the required analytic extension of the zeta function to the real part greater than zero and Euler product was used to prove the real part of the complex that are less than zero and greater or equal to one. Results: From this proposed study we noted that the real values of the complex variable are lying between zero and one which may help to understand the relation between zeta function and its properties and consequently can pay the way to solve some complex arithmetic problems including the Riemann Hypothesis. Conclusion: The combination of Abel transformation and Euler product is a powerful tool for proving theorems and functions related to Zeta function including other subjects such as radio atmospheric occultation.
\end{abstract}

Key words: Zeta function, Abel transformation, Euler product

\section{INTRODUCTION}

There are a number of mathematical functions called "Zeta function" named for their customary symbol, the Greek letter $\zeta$. Of them all, the most famous is the Riemann Zeta Function, for its involvement in the Riemann Hypothesis, which is highly important in Prime Number Theory (PNT). The Riemann Zeta function $\zeta(s)$ is the function of a complex variable $s$ initially defined by the following infinite series:

$$
\xi(s)=\sum_{n=1}^{\infty} \frac{1}{n^{s}}
$$

For values of s with real part greater than one and then analytically continued to all complex with $\mathrm{s} \neq 1$.

Where:

$$
\mathrm{n}^{\mathrm{s}}=\mathrm{e}^{\mathrm{zln} n}, \text { since }\left|\mathrm{n}^{\mathrm{s}}\right|=\mathrm{n}^{\mathrm{Rez}}
$$

This Dirichlet series converges for all real values of s greater than one. Since the 1859 study of Bernhard Riemann, (Castellanos, 1988; David, 1998), it has become standard to extend the definition of $\zeta(\mathrm{s})$ to a complex values $s$; by showing that the series is converges for all complex $\mathrm{s}$ whose real part $\operatorname{Re}(\mathrm{z})$ is greater than one and defines an analytic function of the complex variable $s$ in the region $\{z \in C: \operatorname{Re}(z)>1\}$ of complex plane (Fig. 1).

As a result, the Zeta function becomes an holomorphic in the region $\{\mathrm{z} \in \mathrm{C}: \mathrm{z} \neq 1\}$ of the complex plane and has a simple pole at $\mathrm{s}$ with residue $=1$. The connection between the Zeta function and prime numbers was discovered by Leonhard Euler who proved the identity (Choudhury, 1995) where, by definition, the left hand side is $\zeta(\mathrm{s})$ and the infinite product in the right hand side extend s over all prime $\mathrm{p}$. Thus the expression is called Euler product:

$$
\sum_{\mathrm{n}=1}^{\infty} \frac{1}{\mathrm{n}^{\mathrm{s}}}=\prod_{\text {prime }}\left(\frac{1}{1-\mathrm{p}^{-\mathrm{s}}}\right)
$$

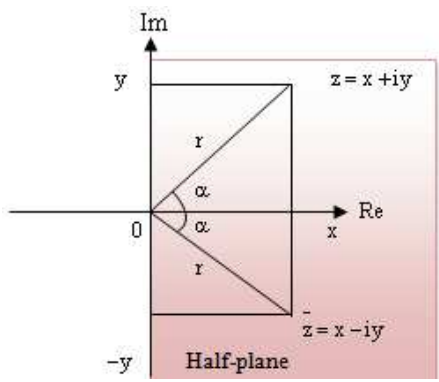

Fig. 1: $\mathrm{Z}$ and its conjugate $\bar{Z}$ in the complex plane 
Here $\mathrm{n}$ ranges over all positive integers $(\mathrm{n}=1,2,3$, $4, \ldots)$ and $\mathrm{p}$ ranges over all primes $(\mathrm{p}=2,3,5,7, \ldots)$. This formula, which is now know as the Euler product results from expanding each of the factors on the right

$$
\left(\frac{1}{1-\mathrm{p}^{-\mathrm{s}}}\right)=1+\frac{1}{\mathrm{p}^{\mathrm{s}}}+\frac{1}{\left(\mathrm{p}^{2}\right)^{\mathrm{s}}}+\frac{1}{\left(\mathrm{p}^{3}\right)^{\mathrm{s}}}+\cdots \cdot
$$

And observing that their product is therefore a sum of term of the form:

$$
\frac{1}{\left(p_{1}^{n 1} p_{2}^{n 2} p_{3}^{n 3} \cdots \cdots p_{r}^{n n}\right)^{z}}
$$

Where:

$$
\begin{aligned}
& \mathrm{p}_{1}, \cdots \cdots \cdot, \mathrm{p}_{\mathrm{r}}=\text { Distinct prime } \\
& \mathrm{n}_{1}, \cdots \cdots \cdot, \mathrm{n}_{\mathrm{r}}=\text { Natural numbers }
\end{aligned}
$$

and then using the fundamental theorem of arithmetic, that is to say every integer can be written in essentially only one way as a product of primes to conclude that the sum is simply:

$$
\sum \frac{1}{n^{s}}
$$

Euler used this formula principally as a formal identity and principally for integer values of s. Dirichlet (Ohtsuka,1967) also based his work in this field on the Euler product formula since Dirichlet was one of Riemann's teacher and since Riemann refers to Dirichlet's work in the first paragraph of his study. It seems certain that Riemann's use of the Euler formula was influenced by Dirichlet. Dirichlet, unlike Euler, used formula (2) with $\mathrm{s}$ as a real variable and, also unlike Euler he proposed rigorously that (1) is true for all real $s>1$. Riemann, as one of the founders of the theory of function of a complex variable, would naturally be expected to consider $\mathrm{s}$ as a complex variable. It is easy to show that both sides of the Euler product formula converge for complex $\mathrm{s}$ in the half plane $\operatorname{Re}(z)>1$, but Riemann goes much further and shows that even though both sides of (2) diverge for other values of $\mathrm{s}$, the function they define is meaningful for all values of $\mathrm{s}$ expect for the pole at $\mathrm{s}=1$.

\section{MATERIALS AND METHODS}

Extension theorem for zeta: The function $\varsigma(z)-\frac{1}{z-1}$ has an analytic extension to the right half plane
$\operatorname{Re}(z)>0$. Thus $\varsigma$ has an analytic to $\{z: \operatorname{Re}(z)>0, z \neq 1\}$ and has a simple pole with residue 1 and $z=1$.

Proof: To prove this extension theorem we used the Abel transformation generally known as summation by parts:

$\sum_{\mathrm{k}=\mathrm{r}}^{\mathrm{s}} \mathrm{a}_{\mathrm{k}} \Delta \mathrm{b}_{\mathrm{k}}=\mathrm{a}_{\mathrm{s}+1} \mathrm{~b}_{\mathrm{s}+1}-\mathrm{a}_{\mathrm{r}} \mathrm{b}_{\mathrm{r}}-\sum_{\mathrm{k}=\mathrm{r}}^{\mathrm{s}} \mathrm{b}_{\mathrm{k}} \Delta \mathrm{a}_{\mathrm{k}}$

With:

$\Delta \mathrm{b}_{\mathrm{k}}=\mathrm{b}_{\mathrm{k}+1}-\mathrm{b}_{\mathrm{k}}$

Where:

$\mathrm{r}=$ Radius of the complex variable

$\mathrm{s}=$ Simple pole

Abel transformation is the discrete analogue of the formula for integration by parts of the form $u(x) d v(x)$ by an integral of $v(x) d u(x)$. For a definite integral the formula of integration by parts is:

$\int_{a}^{b} u(x) d v(x)=u(b) v(b)-u(a) v(a)-\int_{a}^{b} v(x) d u(x)$

This integration by parts is applied under the assumptions that $\mathrm{u}, \mathrm{v}$ and their derivation $\mathrm{u}^{\prime}, \mathrm{v}$ ' are continuous on $a \leq x \leq b$. The (8) is valid whenever both $u$ and $\mathrm{v}$ are absolutely continuous on the closed interval $[a, b]$. Abel transformation is often used to prove many criteria of convergence of series of number and functions. Abel transformation of a series often yields a series with and identical sum, but with a better convergence.

Substituting (7) into (6) we obtain:

$$
\begin{aligned}
& \sum_{\mathrm{k}=\mathrm{r}}^{\mathrm{s}} \mathrm{a}_{\mathrm{k}} \Delta\left(\mathrm{b}_{\mathrm{k}+1}-\mathrm{b}_{\mathrm{k}}\right)=\mathrm{a}_{\mathrm{s}+1} \mathrm{~b}_{\mathrm{s}+1}-\mathrm{a}_{\mathrm{r}} \mathrm{b}_{\mathrm{r}}-\sum_{\mathrm{k}=\mathrm{r}}^{\mathrm{s}} \mathrm{b}_{\mathrm{k}} \Delta \mathrm{a}_{\mathrm{k}} \\
& =\sum_{\mathrm{k}=\mathrm{r}}^{\mathrm{s}} \mathrm{a}_{\mathrm{k}} \Delta \mathrm{b}_{\mathrm{k}+1}-\sum_{\mathrm{k}=\mathrm{r}}^{\mathrm{s}} \mathrm{a}_{\mathrm{k}} \Delta \mathrm{b}_{\mathrm{k}} \\
& =\mathrm{a}_{\mathrm{s}+1} \mathrm{~b}_{\mathrm{s}+1}-\mathrm{a}_{\mathrm{r}} \mathrm{b}_{\mathrm{r}}-\sum_{\mathrm{k}=\mathrm{r}}^{\mathrm{s}} \mathrm{b}_{\mathrm{k}} \Delta \mathrm{a}_{\mathrm{k}}
\end{aligned}
$$

For $\operatorname{Re}(z)>1$ and in order to apply the Abel transformation we suppose that:

$$
\mathrm{a}_{\mathrm{k}}=\mathrm{n}
$$

and:

$$
b_{k}=1 / n^{z}
$$


Then substituting them into (6) we get the following expression:

$$
\sum_{k=1}^{k-1} n\left[\frac{1}{(n+1)^{z}}-\frac{1}{n^{z}}\right]=\frac{1}{n^{z-1}}-1-\sum_{k=1}^{k-1} \frac{1}{(n+1)^{z}}
$$

Were:

$\mathrm{r}=1$

$\mathrm{s}=\mathrm{k}-1$ respectively

Thus:

$$
\left\{\begin{array}{l}
{\left[\begin{array}{c}
\frac{1}{n^{z-1}}+1+ \\
\left.\sum_{n=1}^{k-1} \frac{1}{(n+n)^{z}}\right]
\end{array}\right]=\sum_{n=1}^{k-1}\left[\frac{1}{(n+1)^{z}}-\frac{1}{n^{z}}\right]} \\
{\left[\begin{array}{l}
\frac{1}{n^{z-1}}+1+ \\
\left.\sum_{n=1}^{k-1} \frac{1}{(n+n)^{z}}\right]
\end{array}\right]=-\sum_{n=1}^{k-1}\left[\frac{1}{(n+1)^{z}}-\frac{1}{n^{z}}\right]} \\
1+\sum_{n=1}^{k-1} \frac{1}{(n+n)^{z}}=\frac{1}{n^{z-1}}-\sum_{n=1}^{k-1} n\left[\begin{array}{c}
\frac{1}{(n+1)^{z}} \\
-\frac{1}{n^{z}}
\end{array}\right]
\end{array}\right.
$$

When seeing the second term of the right hand side of (11), it represents the primitive of the following integral:

$$
n x \int_{n}^{n+1} \frac{x}{z+1} d x n=x \int_{n}^{n+1}[x] \frac{x}{z+1} d x
$$

That is to say:

$$
\begin{aligned}
\mathrm{n}\left[\frac{1}{(\mathrm{n}+1)^{\mathrm{z}}}-\frac{1}{\mathrm{n}^{\mathrm{z}}}\right] & =\mathrm{nx} \int_{\mathrm{n}}^{\mathrm{n}+1} \frac{\mathrm{x}}{\mathrm{z}+1} \mathrm{dxn} \\
& =\mathrm{x} \int_{\mathrm{n}}^{\mathrm{n}+1}[\mathrm{x}] \frac{\mathrm{x}}{\mathrm{z}+1} \mathrm{dx}
\end{aligned}
$$

where, $[\mathrm{x}]$ is largest integer less than or equal to $\mathrm{x}$.

Inserting (13) in to the second term of (11) we have:

$$
\begin{aligned}
\sum_{n=1}^{k} \frac{1}{n^{z}} & =1+\sum_{n=1}^{k-1} \frac{1}{(n+1)^{z}} \\
& =\frac{1}{k^{z-1}}+z \sum_{n=1}^{k-1} \int_{n}^{k-1}[x] \frac{x}{z+1} d x
\end{aligned}
$$

Now, letting $\mathrm{x} \rightarrow \infty$ and using the convention that: $\left\{\begin{array}{l}\frac{1}{0}=\infty \\ \frac{1}{\infty}=0\end{array}\right.$

We obtain the following integral for $\operatorname{Re}(z)>1$ :

$$
\zeta(\mathrm{z})=\mathrm{z} \int_{1}^{\infty}[\mathrm{x}] \frac{\mathrm{x}}{\mathrm{z}+1} \mathrm{dx}
$$

Consider the closely related integral:

$$
\begin{aligned}
& \mathrm{z} \int_{1}^{\infty} \mathrm{x} \frac{\mathrm{x}}{\mathrm{z}+1} \mathrm{dx}=\mathrm{z} \int_{1}^{\infty} \frac{\mathrm{x}}{\mathrm{z}} \mathrm{dx}=\mathrm{z}\left[\frac{\mathrm{x}^{1-\mathrm{z}}}{1-\mathrm{z}}\right]_{1}^{\infty} \\
& =\mathrm{z}\left[0-\frac{1}{1-\mathrm{z}}\right]=\frac{\mathrm{z}}{\mathrm{z}-1}=1+\frac{1}{\mathrm{z}-1}
\end{aligned}
$$

Combining (15) and (16), we can write the definition of the extension theorem as follow:

$$
\zeta(\mathrm{z})-\frac{1}{\mathrm{z}-1}=1+\mathrm{z} \int_{1}^{\infty}([\mathrm{x}]-\mathrm{x}) \frac{\mathrm{x}}{\mathrm{z}+1} \mathrm{dx}
$$

Now, if we fix $\mathrm{k}>1$ and consider the integral:

$\int_{1}^{k}([x]-x) \frac{x}{z+1} d x$

We can see that this integral is an analytic of $\mathrm{Z}$ and if $\operatorname{Re}(\mathrm{z})>1$, then:

$\left|\int_{1}^{k}([x]-x) \frac{x}{z+1}\right| \leq \int_{1}^{k} x^{\operatorname{Re}(z+1)} d x$

$\leq \int_{1}^{\mathrm{k}} \frac{\mathrm{x}}{1-\operatorname{Re}(\mathrm{z})} \mathrm{dx}=\frac{1}{\operatorname{Re}(\mathrm{z})}$

This integral implies that the sequences:

$$
\int_{k}(z)=\int_{1}^{k}([x]-x) \frac{x}{z+1} d x
$$

Of an analytic on real part of the complex $z$ is greater than zero that is to say $\operatorname{Re}(z)>0$ is uniformly bounded on compact subsets. Using the Vitali theorem, let $\left[b_{n}\right]$ be a bounded sequence in $A(\Omega)$.

Where:

$\Omega=$ connected

Supposed that $\left[f_{n}\right]$ converges for point wise on $S \subseteq \Omega$ and $S$ has a limit point $\Omega$. Then $\left[f_{n}\right]$ is uniformly 
Cauchy on compact subsets of $\Omega$, hence uniformly converge in compact subsets of $\Omega$ respectively.

Hence:

$$
\int_{k}(z)=\int_{1}^{k}([x]-x) \frac{x}{z+1} d x
$$

is analytic and thus the function:

$$
1+\mathrm{z} \int_{1}^{\infty}([\mathrm{x}]-\mathrm{x}) \frac{\mathrm{x}}{\mathrm{z}+1} \mathrm{dx}
$$

is analytic on $\operatorname{Re}(\mathrm{z})>0$. But this function agrees with $\zeta(\mathrm{z})-\frac{1}{\mathrm{z}-1}$ for $\operatorname{Re}(\mathrm{z})>1$ and consequently provides the required analytic extension of $\xi$ to $\operatorname{Re}(z)>0$. Hence the proof of the extension theorem is completed. We have seen that Euler product implies that $\xi$ has no zeros in the half plane $\operatorname{Re}(\mathrm{z})>1$. But how about zeros of the extension of $\xi$ in $0<\operatorname{Re}(\mathrm{z}) \leq 1$. The next theorem asserts tells that $\xi$ has no zeros on the line $\operatorname{Re}(\mathrm{z})=1$.

Theorem: For $\operatorname{Re}(\mathrm{z})>1$ :

$\frac{\zeta^{\prime}(\mathrm{z})}{\zeta(\mathrm{z})}=\mathrm{z} \int_{1}^{\infty} \frac{\psi(\mathrm{t})}{\mathrm{t}^{\mathrm{z}+1}} \mathrm{dt}$

Where:

$\psi(\mathrm{t})=\sum_{\mathrm{k} \leq \mathrm{x}} \Lambda(\mathrm{n})$

With:

$\Lambda(n)=\left\{\begin{array}{l}\operatorname{lnp} \text { if } n=p^{m} \text { for some } m \\ 0 \text { otherwise }\end{array}\right.$

Where:

$$
\Lambda(\mathrm{n})=\ln \mathrm{p}
$$

if $\mathrm{n}$ is a power of the prime $\mathrm{p}$ and 0 if not. An equivalent expression of $\psi$ is:

$$
\psi(\mathrm{x})=\sum_{\mathrm{p} \leq \mathrm{x}} \mathrm{mp}(\mathrm{x}) \ln \mathrm{p}
$$

where the sum is over the prime $\mathrm{p} \leq \mathrm{x}$ and $\mathrm{m}_{\mathrm{p}}(\mathrm{x})$ is the largest integer such that $\mathrm{p}_{\mathrm{p}}^{\mathrm{m}}{ }^{(\mathrm{x})} \leq \mathrm{x}$.

For example: $\psi(10.4)=3 \ln 2+2 \ln 3+\ln 5+\ln 7$

Note that:

$\mathrm{p}^{\mathrm{m}_{\mathrm{p}}(\mathrm{x})} \leq \mathrm{x}$ iff $\mathrm{m}_{\mathrm{p}}(\mathrm{x}) \ln \mathrm{p} \leq \ln \mathrm{x}$ iff $\mathrm{m}_{\mathrm{p}}(\mathrm{x}) \leq \frac{\ln \mathrm{x}}{\ln p}$

Thus:

$$
\mathrm{m}_{\mathrm{p}}(\mathrm{x})=\left\lfloor\frac{\ln \mathrm{x}}{\ln \mathrm{p}}\right\rfloor
$$

where, $L\rfloor$ is the greatest integer function.

The function $\psi$ will be used to obtain the desired integral representation $\frac{\zeta^{\prime}}{\zeta}$.

Proof: Throughout the study $\mathrm{p}$ and $\mathrm{q}$ range over primes if $\operatorname{Re}(\mathrm{z})>1$. The Riemann zeta function:

$\zeta(\mathrm{z})=\sum_{\mathrm{n}=1}^{\infty} \frac{1}{\mathrm{n}^{2}}$

is given by the Euler product:

$\zeta(\mathrm{z})=\prod_{\mathrm{j}=1}^{\infty}\left(\frac{1}{1-\mathrm{p}_{\mathrm{j}}^{-\mathrm{z}}}\right)=\zeta(\mathrm{z}) \frac{1}{\left(1-\mathrm{p}_{\mathrm{j}}^{-\mathrm{z}}\right)}=\prod_{\mathrm{j}=1}^{\infty}$

Where:

$\mathrm{p}_{\mathrm{j}}=$ Increasing sequence of prime numbers and the product converge uniformly on compact subset of real part is greater than one

$\xi=$ Analytic on $\operatorname{Re}(\mathrm{z})>1$

Furthermore the product of $\xi$ shows that $\xi$ has no zeros in $\operatorname{Re}(\mathrm{z})>1$.

Hence:

$\frac{\zeta^{\prime}(\mathrm{z})}{\zeta(\mathrm{z})}=\sum_{\mathrm{p}} \frac{-\mathrm{p}^{-\mathrm{z}} \ln \mathrm{p}}{\left(1-\mathrm{p}^{-\mathrm{z}}\right)^{2}} \Rightarrow \zeta^{\prime}(\mathrm{z})=\sum_{\mathrm{p}} \frac{-\mathrm{p}^{-\mathrm{z}} \ln \mathrm{p}}{\left(1-\mathrm{p}^{-\mathrm{z}}\right)^{2}} \zeta(\mathrm{z})$

Substituting (26) into (27) we have:

$\zeta^{\prime}(\mathrm{z})=\sum_{\mathrm{p}} \frac{-\mathrm{p}^{-\mathrm{z}} \ln \mathrm{p}}{\left(1-\mathrm{p}^{-\mathrm{z}}\right)^{2}} \prod_{\mathrm{p} \neq \mathrm{q}}\left(\frac{1}{1-\mathrm{p}^{-\mathrm{z}}}\right)$

Where:

$\prod_{\mathrm{j}=1}^{\infty}=\zeta(\mathrm{z}) \frac{1}{\left(1-\mathrm{p}_{\mathrm{j}}^{-\mathrm{z}}\right)}$ 
Equation 29 becomes:

$$
\begin{aligned}
& \zeta^{\prime}(\mathrm{z})=\sum_{\mathrm{p}} \frac{-\mathrm{p}^{-\mathrm{z}} \ln \mathrm{p}}{\left(1-\mathrm{p}^{-\mathrm{z}}\right)^{2}} \zeta(\mathrm{z})\left(\frac{1}{1-\mathrm{p}^{-\mathrm{z}}}\right) \frac{1}{\left(1-\mathrm{p}^{-\mathrm{z}}\right)} \\
& =\zeta(\mathrm{z}) \frac{-\mathrm{p}^{-\mathrm{z}} \ln \mathrm{p}}{\left(1-\mathrm{p}^{-\mathrm{z}}\right)^{2}}\left(1-\mathrm{p}^{-\mathrm{z}}\right)^{0}
\end{aligned}
$$

Using the convention that $\mathrm{a}^{0}=1$, we have:

$$
\zeta^{\prime}(\mathrm{z})=\zeta(\mathrm{z}) \sum_{\mathrm{p}} \frac{-\mathrm{p}^{-\mathrm{z}} \ln \mathrm{p}}{\left(1-\mathrm{p}^{-\mathrm{z}}\right)^{2}}=\frac{\zeta^{\prime}(\mathrm{z})}{\zeta^{\prime}(\mathrm{z})}=\sum_{\mathrm{p}} \sum_{\mathrm{n}=1} \mathrm{p}^{-\mathrm{nz}} \ln \mathrm{p}
$$

As the iterated sum of (33) is absolutely convergent for $\operatorname{Re}(\mathrm{z})>1$, we can rearrange it as a double sum as:

$$
\sum_{p_{n}(n>1)}\left(p^{n}\right)^{-z} \ln p=\sum_{k} k^{-z} \ln p
$$

where, $\mathrm{p}^{\mathrm{n}}$ is $\mathrm{k}$ for sum $\mathrm{n}$.

Consequently (33) becomes:

$$
\frac{\zeta^{\prime}(\mathrm{z})}{\zeta^{\prime}(\mathrm{z})}=\sum_{\mathrm{k}=1}^{\infty} \mathrm{k}^{-\mathrm{z}} \Lambda(\mathrm{k})=\sum_{\mathrm{k}=1}^{\infty} \mathrm{k}^{-\mathrm{z}}(\psi(\mathrm{k})-\psi(\mathrm{k}-1))
$$

By the definition of $\Lambda$ and $\psi$. But using Abel transformation once again with:

$$
\left\{\begin{array}{l}
a_{k}=k^{-z} \\
b_{n+1}=\psi(k-1) \\
b_{1}=\psi(0) \text { and } M=\infty
\end{array}\right.
$$

We have:

$$
\begin{aligned}
& \sum_{\mathrm{k}=1}^{\infty} \mathrm{k}^{-\mathrm{z}} \psi(\mathrm{k})-\psi(\mathrm{k}-1)=\psi(\mathrm{M})(\mathrm{M}+1)^{-1} \\
& +\sum_{\mathrm{k}=1}^{\infty} \mathrm{k}^{-\mathrm{z}} \psi(\mathrm{k})\left[\mathrm{K}^{-\mathrm{z}}-(\mathrm{k}+1)^{-\mathrm{z}}\right]
\end{aligned}
$$

Now from the definition of:

$$
\psi(x)=\sum_{n \leq x} \Lambda(n)
$$

We obtain $\psi(x) \leq x \operatorname{Inx}, \quad$ so if $\operatorname{Re}(\mathrm{z})>1$ we have $\frac{\psi(\mathrm{M})(\mathrm{M}+1)^{-\mathrm{z}}}{\mathrm{z}} \rightarrow 0$ and as $\mathrm{M} \rightarrow \infty$, we can write:

$$
\begin{aligned}
& \sum_{\mathrm{k}=1}^{\mathrm{M}} \psi(\mathrm{k})\left(\frac{\mathrm{K}}{\mathrm{z}}-\frac{(\mathrm{k}+1)}{\mathrm{z}}\right)=\sum_{\mathrm{k}=1}^{\mathrm{M}} \psi(\mathrm{k}) \mathrm{z} \int_{\mathrm{k}}^{\mathrm{k}+1} \frac{\mathrm{t}}{\mathrm{z}+1} \mathrm{dt} \\
& =\sum_{\mathrm{k}=1}^{\mathrm{M}} \mathrm{z} \int_{\mathrm{k}}^{\mathrm{k}+1} \psi(\mathrm{k}) \frac{\mathrm{t}}{\mathrm{z}+1} \mathrm{dt}=\mathrm{z} \int_{1}^{\mathrm{M}} \psi(\mathrm{t}) \frac{\mathrm{t}}{\mathrm{z}+1} \mathrm{dt}
\end{aligned}
$$

Because $\psi$ is constant in each interval [k, $\mathrm{k}+1]$. Taking the limit of (38) if $\mathrm{M} \rightarrow \infty$ we have finally:

$$
\frac{\zeta^{\prime}(\mathrm{z})}{\zeta(\mathrm{z})}=\mathrm{z} \int_{1}^{\mathrm{M}} \psi(\mathrm{t}) \frac{\mathrm{t}}{\mathrm{z}+1} \mathrm{dt} \text { for } \operatorname{Re}(\mathrm{z})>1
$$

The proof is completed.

\section{RESULTS}

In this study we have learned three important things:

- The first thing is that the Riemann zeta function

$$
\zeta(\mathrm{z})=\sum_{\mathrm{n}=1}^{\infty} \frac{1}{\mathrm{n}^{\mathrm{z}}}
$$

Which is given by the following product:

$$
\prod_{j=1}^{\infty}\left(\frac{1}{1-p_{j}^{-z}}\right)
$$

where, $P_{j}$ is sequence of prime numbers converge uniformly on compact subsets of $\operatorname{Re}(\mathrm{z})>1$, hence $\xi$ is analytic on $\operatorname{Re}(\mathrm{z})>1$. Furthermore we have noted also that the Euler product representation of $\xi$ shows that $\xi$ has no zeros in $\operatorname{Re}(\mathrm{z})>1$ and consequently it can be extended to a region larger than $\operatorname{Re}(\mathrm{z})>1$

- The second thing is the zeta's logarithmic derivative $\xi^{\prime}(\mathrm{z}) / \xi(\mathrm{z})$ we have used to prove that $\xi$ can have some value in $0<\operatorname{Re}(\mathrm{z}) \leq 1$, which we know is analytic on $\operatorname{Re}(\mathrm{z})>1$. In fact the true is that $\xi^{\circ}(\mathrm{z}) / \xi(\mathrm{z})$ is analytic only on a neighborhood of $\{Z: \operatorname{Re}(z)>1$ and $Z \neq 1\}$. Since $\xi$ has a simple pole at $\mathrm{z}=1$ so does $\xi^{\prime}(\mathrm{z}) / \xi(\mathrm{z})$, with residue $\operatorname{Res}\left(\xi^{\prime}(\mathrm{z}) / \xi(\mathrm{Z}), 1\right)=-1$. We next obtain an integral representation for $\xi^{\circ}(\mathrm{z}) / \xi(\mathrm{z})$ that is similar to representation (15) of $\xi$

- The function $\psi$ define in (21) provides another connection through (22) between the Riemann zeta function and the properties of the prime numbers. The integral that appear in (21) is called Mellin transform of $\psi$ 


\section{DISCUSSION}

The combination of Abel transformation (Camelia and Gruia, 2008) and Euler product has helped us to understand the relation between zeta function and its proprieties which may pay the way to solve some complex theorems (Weimann, 2007) and arithmetic or geometric problems including problems related to radio atmospheric occultation. For example: Thomson (2007) proposed in his research that radio occultation measurements made with a receiver inside the earth's atmosphere can be inverted with an Abel transform to provide an estimate of atmospheric refractive index profile. Healy et al. (2002), also proposed in their study that the reformulation of the radon transform as path integral for the case of a radio ray refracting in a spherical symmetric atmosphere can be change to Abel transformation as both are equivalent in atmospheric radio occultation and $\mathrm{I}$ agree with their proposed theory. Some successful work related to Riemann zeta function and can be found in: Choudhury (1995); Conrey (2003) and Cvijovic and Klinowski (2002).

\section{CONCLUSION}

In this study, we have prove the extension theorem fro zeta function based ion Abel transformation and Euler product where the Abel transformation has been used to prove the real values of the complex variable that are only greater zero and next the Euler product is used to deal with the real values less than zero and greater or equal to one. The proposed study has helped us not only to understand the relation between zeta function and its properties but also shows the function $\psi$ defines in (21) provides another connection through (22) between Riemann zeta function and properties of the prime numbers.

\section{REFERENCES}

Camelia, G. and I. Gruia, 2008. Solving the integral equation of the inverse Abel transform using cubic spline interpolation application to plasma spectroscopy. Escampig. http://www.escampig2008.csic.es/PosterSessions/8 3.pdf
Castellanos, D., 1988. The ubiquitous Pi. Math. Mag., 61: 148-163. http://www.dm.unito.it/ cerruti/ac/piubi-2.pdf

Choudhury, B.K., 1995. The Riemann zeta-function and its derivatives. Proc. Roy. Soc., 450: 477-499. DOI: 10.1098/rspa.1995.0096

Conrey, J.B., 2003. The Riemann hypothesis. Notices Am. Math. Soc., 50: 341-353. http://www.ams.org/notices/200303/fea-conreyweb.pdf

Cvijovic, D. and J. Klinowski, 2002. Integral representations of the riemann zeta function for odd-integer arguments. J. Comput. Applied Math., 142: 435-439.

David, R.W., 1998. On the number of prime numbers less than a given quantity. Monatsberichte der Berliner Akademie. http://www.maths.tcd.ie/pub/HistMath/People/Rie mann/Zeta/EZeta.pdf

Healy, S.B., J. Haase and O. Lesme, 2002. Abel transformation inversion of radio occultation measurements made with a receiver inside the eart's atmosphere. J. Eur. Geophys. Soc., 20: 1253-1256. http://www.anngeophys.net/20/1253/2002/angeo-20-1253-002.pdf

Ohtsuka, M., 1967. Dirichlet principle on Riemann surfaces. J. D'anal. Math., 19: 295-311. DOI: 10.1007/BF02788723

Thomson, F.S., 2007. Radon and Abel transform equivalence atmospheric radio occultation. Radio Sci., 42: 3024-3026. DOI: 10.1029/2006RS003583

Weimann, M., 2007. Concavity, Abel transform and the Abel inverse theorem in smooth complete toric varieties; Joseph Fourier University. http://arxiv.org/abs/0705.0247 